Revue musicale OICRM

\title{
Rire, mémoire, Shoah, d'Andréa Lauterwein (dir.)
}

\section{Gabriel Paquin-Buki}

Volume 3, numéro 2, 2016

Mémoire musicale et résistance. Autour du Verfügbar aux Enfers de Germaine Tillion

URI : https://id.erudit.org/iderudit/1060112ar

DOI : https://doi.org/10.7202/1060112ar

Aller au sommaire du numéro

Éditeur(s)

OICRM

ISSN

2368-7061 (numérique)

Découvrir la revue

Citer ce compte rendu

Paquin-Buki, G. (2016). Compte rendu de [Rire, mémoire, Shoah, d’Andréa

Lauterwein (dir.)]. Revue musicale OICRM, 3(2), 157-164.

https://doi.org/10.7202/1060112ar d'utilisation que vous pouvez consulter en ligne.

https://apropos.erudit.org/fr/usagers/politique-dutilisation/ 


\section{Rire, mémoire, Shoah, d'Andréa Lauterwein (dir.) Paris, Éditions de l'Éclat, 2009, 400 pages}

\section{Gabriel Paquin-Buki}

Mots clés : humour ; mémoire ; rire ; Shoah ; transmission.

Keywords: humor; memory; laughter; Shoah; transmission.

Ya-t-il un rire après la Shoah ? Non ! Du moins pas chez le dentiste ${ }^{1}$ !

Rire, Mémoire, Shoah

ouvrage coordonné par Andréa Louterwein avec la colloboration de Colette Strouss-Hivo

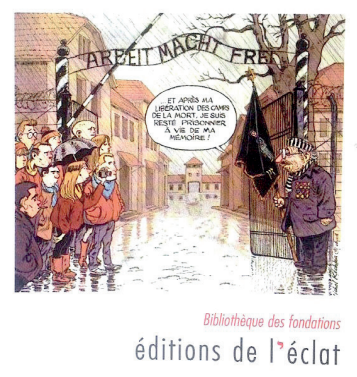

éditions de l'éclat

Paru en 2009, l'ouvrage collectif Rire, mémoire, Shoah est dirigé par Andréa Lauterwein, professeure au Département d'études germaniques à l'Université Sorbonne-Nouvelle, spécialiste de Paul Celan et d'Anselm Kiefer, et auteure notamment du livre Essai sur la mémoire de la Shoah en Allemagne fédérale (Paris, Kimé, 2005). Le collectif a été réalisé avec la collaboration de Colette Strauss-Hiva, professeure à l'Institut d'études politiques de Paris, spécialiste de la littérature allemande contemporaine et traductrice d'écrivains germanophones contemporains (notamment Zafer Şenocak et Doron Rabinovici). Les 400 pages du volume contenant les textes ou entretiens de 25 auteurs différents représentent le travail le plus vaste ayant été réalisé jusqu'à maintenant sur la question du rire et de la Shoah, bien que les deux 
collaboratrices soient conscientes du fait que leur étude reste encore incomplète. En effet, elles tiennent à préciser - sous forme d'avertissement - que le livre constitue une sorte de balisage, un " matériau à penser » (p. 6).

Bien que le titre du livre puisse sembler provocateur, par la juxtaposition des termes " rire » et "Shoah », il ne faut pas y voir un désir de polémique, mais plutôt de débat sur la nature du rire qui s'est immiscé dans la question juive et sur la manière dont celui-ci a pu façonner la transmission de la mémoire des années 1930 jusqu'au $\mathrm{XXI}^{\mathrm{e}}$ siècle. La question du rire, par sa traditionnelle association au bonheur ou à la légèreté, aura mis plusieurs décennies avant d'être considérée comme une approche potentielle $\mathrm{du}$ génocide. Mais quand finalement on jugea le rapprochement entre l'humour et la Shoah "de son temps ", les questions étaient nombreuses, comme en témoigne Lauterwein dans son introduction :

L'ambivalence intrinsèque du rire contribue-t-elle à soutenir une culture du dissensus, salutaire pour la perpétuation de la mémoire? Comment faire face à l'indifférence des générations futures sans pour autant les entraîner dans une névrose de culpabilité ? [...] Le rire peut-il libérer certains affects bloqués, pour engager à un travail de mémoire ? [...] Le rire, comme expérience physique de l'effondrement, peut-il éventuellement abattre les barrières qui cloisonnent les mémoires individuelles (p. 5) ?

Ces questions ne sont certes pas spécifiques à la Shoah. Toutefois, l'ampleur de la Catastrophe fera du génocide juif une sorte de référence. De plus, il ne faut pas négliger le rôle de la longue tradition d'humour juif dans la possibilité même d'une telle étude, bien que Rire, mémoire, Shoah traite du rire dans toutes ses manifestations, juives ou non.

Le rire dont il est question ici, prévient l'auteure principale, est un rire « qui rend rarement heureux» (p. 7). On ne rit pas d'Auschwitz, mais bien de ce qu'on désigne par "Auschwitz ", cette métaphore kitsch de l'horreur, cette image aujourd'hui muséifiée présente dans l'imaginaire collectif et qui englobe dans ce simple nom l'ensemble des atrocités perpétrées dans les camps. On rit de ce cliché d'un "Auschwitz » qui, à la fois, a toujours et n'a jamais existé, et qui bloque l'accès à une réelle transmission de la mémoire. Le livre est divisé en trois parties : "Le rire à la limite » (p. 15-118), "Le rire étranglé » (p. 119-250) et "Questions de controverses » (p. 251-394). Pour chacune d'entre elles, nous traiterons en priorité des textes qui reflètent le mieux l'évolution du traitement de la Shoah par le rire.

« LE RIRE À LA LIMITE »

La première partie de Rire, mémoire, Shoah traite des manifestations de certaines formes d'humour, comme la satire et l'autodérision, particulièrement entre 1933, l'année où Hitler a pris le pouvoir, et 1945, qui marque une césure historique. «Le rire à la limite ", c'est le rire de ceux qui ont traversé l'enfer avec cette arme des désarmés. Cette première partie de l'ouvrage est traitée par six auteurs.

Pour Gerald Stieg, Marianne Dautrey et Stephan Braese, l'écrivain juif autrichien Karl Kraus est l'un des représentants de ce rire. Stieg, spécialiste de la littérature 
allemande, des contes et de la littérature jeunesse, décrit la profondeur de la satire de Kraus qui prévoyait la montée du national-socialisme dès les années 1920 et qui l'a décriée jusque dans les années 1930. Selon Stieg, La troisième nuit de Walpurgis, publié en 1933, constitue "un des grands textes-diagnostic du "malaise dans la culture" " (p. 26) et témoigne de la façon dont le régime nazi a pris possession du pouvoir. Marianne Dautrey, traductrice et professeure de philosophie, abonde dans le même sens, écrivant que dans une sorte de " témoignag[e] de ce qui n'avait pas encore eu lieu » (p. 29), Kraus pressentait les horreurs à venir. Rapidement, il a ridiculisé la montée du nazisme, mais sa satire a pris toute sa valeur lorsque le parti fut réellement élu et que les idées qu'il s'amusait à associer au Neandertal devinrent des actions concrètes. Pour Dautrey, la satire de Kraus et l'œuvre de Walter Benjamin sont intimement liées. Le rire du satiriste, elle l'associe à un passage d'Expériences et pauvreté qu'a écrit Benjamin en 1933 : " Dans leurs bâtiments, dans leurs tableaux et leurs récits, l'humanité s'apprête à survivre, s'il le faut, à la culture. Et surtout, elle le fait en riant. Ce rire peut parfois sembler barbare» (p. 31). Kraus était le représentant de ce rire barbare, dans un monde où la satire se confondait avec la réalité, où la caricature devenait la norme. Ne pouvant plus espérer déconstruire l'idéologie nazie, ce rire préparait l'humain "à survivre à la culture et à atteindre un "après" de la culture " (p. 41), note Braese, spécialiste du national-socialisme. La satire de Kraus a jeté un pont entre la génération d' " avant » et celle d' " après ». Mais entre les deux, il peut être difficile de concevoir la place qu'occupait le rire pendant la Shoah. Pourtant, pour les déportés, le rire était devenu une aide précieuse à la survie. Dans son texte « Du rire anti-nazi au rire catastrophé " (p. 79-106), Lauterwein recense ainsi les formes d'humour qui se sont manifestées durant 1'Holocauste et offre une étude poignante sur la façon dont elles ont permis aux détenus des ghettos et des camps de conserver un semblant d'humanité.

Les témoignages des camps montrent bien que la Shoah a donné un sens extrême à plusieurs concepts. Que ce soit la faim, la soif ou la fatigue, rarement ces termes ont-ils eu une signification aussi éloignée de celle qui est sous-entendue dans leur emploi actuel. Il en va de même pour le rire. Lauterwein le rappelle : " dans une société libre, une blague est un luxe» (p. 82), mais pendant la guerre, les effets d'une blague pouvaient jouer un rôle important dans la survie des détenus en créant un lien de solidarité, un outil de résistance et surtout un mécanisme de défense psychologique face à la souffrance et aux humiliations. Pour cette raison, avant d'être définitivement interdits par les nazis en 1941 sur tout leur territoire, les cabarets d'humour étaient très importants. Les contenus qu'ils véhiculaient, faciles à retenir, ont sommeillé dans les trains et firent leur chemin jusque dans les camps, où leurs bienfaits furent considérables.

Le rire permettait aux détenus de garder un lien psychologique avec le genre humain. Traités comme des animaux, toute manifestation de culture leur devenait salutaire. On retrouve ainsi quelques cas de création artistique dans les conditions effroyables des camps, l'un des plus surprenants étant celui du Verfügbar aux Enfers, opérette-revue rédigée par Germaine Tillion à Ravensbrück. Lauterwein décrit la force d'un tel document qui, grâce à sa structure et à ses rimes, sollicitait immanquablement la mémoire, préparant ainsi les détenues à témoigner après la guerre. Plus 
encore, l'autodérision créait une distance entre la détenue et sa propre représentation, provoquant le choc nécessaire à une réaction d'autodéfense ${ }^{2}$.

Mais qu'y a-t-il après l'autodérision, quand les souffrances se font pires de jour en jour ? Il y a ce rire à la limite, ce que Lauterwein appelle le rire catastrophé, dénué d'humour, qui est l' « ultime affirmation de l'existence, au-delà du bien et du mal, [un rire qui] mime l'effondrement du sens par l'effondrement physique qu'il entraîne " (p. 106). Jusqu'à la démence ou la mort, quand toute valeur humaine semble avoir disparu, il semble qu'une forme de rire puisse encore accompagner l'être humain.

\section{« LE RIRE ÉTRANGLÉ »}

Dès 1945, la Shoah a constitué une césure historique et le monde allait dorénavant vivre dans 1'« après ». L'ère et l'art post-Shoah ont d'emblée été marqués par le célèbre diktat d'Adorno : "Écrire un poème après Auschwitz est barbare " (Adorno, "Kulturkritik und Gesellschaft », cité p. 51). En effet, se consacrer à la création artistique après la mort de millions d'humains n'est-il pas aussi une forme de barbarie? Comment donc représenter autre chose que la Catastrophe, elle-même irreprésentable ? Comment le rire peut-il encore s'exprimer lorsque l'humanité a en tête les atrocités perpétrées?

C'est ce rire de la Shoah que Lauterwein qualifie de « rire étranglé », un rire grinçant qui met le lecteur profondément mal à l'aise. Cette deuxième partie de Rire, mémoire, Shoah, qui contient six articles et un entretien, tente de comprendre comment s'est manifesté l'humour dans certains textes post-Shoah et comment ceux-ci outrepassent les tabous.

Dans son article intitulé " Horrible, humour noir, rire blanc » (p. 129-140), la professeure de littérature Judith Kauffmann ${ }^{\dagger}$ différencie le rire qui dénonce (l'humour noir) de celui qui rebâtit (le rire blanc). Elle prend notamment pour exemple La danse de Gengis Cohn (1967) de Romain Gary, où le héros, un ancien comique de cabaret, hante le subconscient de l'officier SS qui l'a exécuté à Auschwitz.

L'humour noir se manifeste à plusieurs reprises dans ce roman, comme lorsque Cohn demande à son voisin de tombeau, juste avant d'être fusillé, ce qu'il pense de la culture, et que celui-ci répond : «La culture, c'est lorsque les mères qui tiennent leurs enfants dans leurs bras sont dispensées de creuser leurs tombes avant d'être fusillées » (p. 137). L'ensemble du roman est truffé de ce genre de déclarations tragi-comiques. Selon Kauffmann, ces mécanismes d'écriture qui feignent de s'approprier le discours du persécuteur permettent d'éviter d'être frappé de plein fouet par l'horreur en l'abordant par une mise à distance dédramatisante.

Le rire blanc est différent. C'est celui du Juif hassid, dont parle Gary, que les Allemands tirent par la barbe et qui sourit avec eux, le regard tourné vers la postérité. C'est un rire de l'autre côté du pont bâti par Karl Kraus, un rire qui reconstruit, un rire « irrémédiablement soudé à l'humain transitoire et dérisoire » (p. 137). Ce type de 
rire est plus rare, mais ô combien important lorsqu'il s'agit d'envisager la possibilité que l'humain puisse rire de nouveau.

Pour Aurélia Kalisky, spécialiste de la littérature du témoignage et notamment de celui des enfants de la Shoah, certains passages de Gengis Cohn ou du Nazi et le barbier (1971), d'Edgar Hilsenrath, écrivain juif allemand de la deuxième génération, se trouvent à mi-chemin entre le noir et le blanc, dans ce qu'elle qualifie de rire gris, qui n'est pas sans rappeler la zone grise dont parle Primo Levi dans Les naufragés et les rescapés (1989), cette zone incertaine qui délimite l'agresseur de l'agressé. En effet, dans son article "Mystères de la satire - Rire gris et humour barbare dans deux romans "après Auschwitz" "(p. 157-176), Kalisky note que dans les deux romans, on retrouve cette situation où l'on ne sait plus distinguer le Juif du nazi, le bourreau de la victime. L'humour sert ici à démasquer la déresponsabilisation dont font preuve les anciens bourreaux qui tendent vers un philosémitisme hypocrite. Ils se fondent aux victimes et leur tendent une main encore tachée de sang. À défaut d'une restauration de la justice, la satire permet celle de l'éthique en ironisant sur l'effacement de la faute et en créant une contre-mémoire. Elle rétablit un semblant de vérité par un mensonge (une fiction) qui en contre un autre (l'hypocrisie).

Pour Lauterwein, les fictions d'Hilsenrath - comme celles de Jurek Becker, écrivain juif allemand, et de Ruth Klüger, écrivaine juive américaine, tous deux de la deuxième génération - sont primordiales pour transmettre une mémoire qui perd peu à peu ses témoins oculaires. Ces auteurs, qui donnent son titre à l'article « Trois passeurs de témoins » (p. 209-242), font moduler la mémoire de factuelle à culturelle. Leur rôle est de réactualiser la mémoire de la Shoah dans l'imaginaire collectif pour que plus jamais ne se reproduise la Catastrophe. Lorsque le témoignage devient impossible, la fiction acquiert une importance capitale dans la transmission de la mémoire et devient plus vraie que l'histoire elle-même, figée dans les livres d'histoire. Dans son article "Dans un instant vous roulerez vers le bonheur » (p. 177-190), Ruth Vogel-Klein, spécialiste des écrivains allemands du $\mathrm{xx}^{\mathrm{e}}$ siècle, explique pourquoi 1'historicisation de la Catastrophe n'est pas souhaitable et montre comment l'humour peut la contrer.

Par l'analyse du roman Un voyage (1962), de H. G. Adler, Vogel-Klein explique brillamment l'effet du rire étranglé sur le lecteur. L'ironie qui peut à tout moment entrecouper les passages sérieux est comparable à un croc-en-jambe qu'on ferait à un marcheur tranquille. Cette forme d'humour sert à "dépouiller de toute assurance " (p. 179) le lecteur qui croit déjà connaître le sentiment qu'il doit éprouver face à la Shoah. L'humour entrave la cristallisation des clichés liés à Auschwitz qui, voulant dénoncer les massacres de façon honnête, font de l'Holocauste un sujet classé qu'il n'est plus besoin de remettre à l'ordre du jour. Or, il semble que pour rester à distance physique de l'homme, l'horreur doive tout au moins rester vivante dans son imaginaire : "L'ironie déréalise, mais rend peut-être encore plus poignante la représentation de l'horreur » (p. 189).

\section{«QUeSTIONS ET CONTROVERSES »}

La troisième et dernière partie de Rire, mémoire, Shoah, qui contient sept articles et quatre entretiens, se consacre à la deuxième et à la troisième générations suivant 
la Shoah. Dès les années 1960, plusieurs écrivains traitant de la Catastrophe étaient aux prises avec une aporie identitaire, séquelle d'un terrible héritage. Ce fut particulièrement le cas chez des auteurs de langue allemande, langue du bourreau, ou chez des écrivains américains, dont le lieu de naissance était loin des massacres. La fin $\mathrm{du} \mathrm{Xx}^{\mathrm{e}}$ siècle fut aussi marquée par un renouveau dans le cinéma comique lié à la Shoah. À travers ces livres et ces films, qu'est-il advenu de la mémoire traumatique? Comment s'est-elle rendue jusqu'à nous, au XXI ${ }^{\mathrm{e}}$ siècle ? Et encore une fois, quelle est la place du rire dans tout cela?

Judith Stora-Sandor, comparatiste et directrice de la revue Humoresques, rappelle dans son article (p. 251-264) que l'humour juif tire ses racines des textes sacrés et que ce trait culturel a toujours caractérisé le peuple juif, à travers ses différentes diasporas. Elle conclut donc : "N'est-ce pas l'âme d'un peuple qui s'exprime dans cet art de vivre et de survivre qu'est son humour? » (p. 264) Ainsi, considérer le peuple juif sans son humour, c'est en quelque sorte lui enlever son âme et son art. À la lumière de cet article, il devient donc plus légitime, voire encourageant de voir l'autodérision, la satire et l'ironie se mêler à la question de la Shoah, même sous la plume d'écrivains n'ayant jamais connu ces atrocités.

C'est par exemple le cas de Robert Schindel, qui truffe ses romans d'humour typiquement juif " permettant à l'auteur de revendiquer une filiation avec la culture disparue » (p. 307), comme en témoigne Béatrice Gonzalés-Vangell, spécialiste de la littérature autrichienne, dans son article «Le rire et la mémoire de la Shoah dans les écrits de Robert Schindel » (p. 301-309). Le rire renoue avec le fil d'Ariane de la culture juive, momentanément perdu de vue, et réinvestit celle-ci par un esprit positif au fond inhérent au rire. L'autodérision, bien qu'autocritique, " est un moyen qui contribue à revaloriser le terme "juif" " (p. 307) dont toute l'histoire s'est teintée de noir au sortir du génocide. Dans un entretien mené par Strauss-Hiva en 2008 (p. 311-322), Schindel indique que la littérature devient pour lui le vecteur d'un « rétablissement d'un continuum historique » (p. 316) lorsqu'elle s'inspire des fondements d'une culture vieille de plusieurs millénaires.

La satire et l'ironie mordantes, qui étaient exceptionnelles chez Gary ou Hilsenrath, sont devenues monnaie courante chez Philip Roth ou Maxim Biller. Ceux-ci cherchent uneéchappatoire au trauma hérité, commeen témoignecepassage extraitdela chronique Le temps des monstres est révolu - écrite par Biller dans les années 1980 - et cité par Manuel Gogos dans son article «Affinités transatlantiques» (p. 265-277) :

Je suis un écrivain juif, je refuse de me faire interdire le rire par les nazis $a$ posteriori. Je veux rire et me sentir mieux après. Je sais combien c'est difficile, combien c'est impossible, car je ne suis pas indifférent au point d'oublier que six millions ont été exterminés pendant la dernière guerre. Quelle merde. Malgré tout, je ne peux pas m'empêcher de faire des blagues hérétiques, je veux enfin me libérer de mon trauma. Une contradiction insurmontable (p. 276).

Cette contradiction est omniprésente dans la littérature post-Shoah. Le trauma, explique Gogos, spécialiste de la littérature juive contemporaine, c'est le statut de victime qui colle à la peau et dont on ne peut se défaire. C'est en quelque sorte l'ombre 
de l'indicible qui se cache derrière toute parole, prête à ressurgir à tout moment. Roth et Biller tentent de briser les paralysies du langage par une écriture souvent violente et provocatrice qui, en déconstruisant les structures usuelles de la mémoire, permettent la création de nouveaux espaces de réflexion. La figure du héros-victime se brise et se transforme en celle d'un intellectuel vivant qui refuse le conformisme étroit par lequel on tend à représenter un Juif chétif et craintif. Suivant l'interprétation de Roth, Kafka n'est plus un cancrelat apeuré, mais plutôt, il « rit de son propre Procès » (p. 277).

Dans un entretien mené par Strauss-Hiva en 2008 (p. 279-289), Doron Rabinovici, écrivain israélo-autrichien de la deuxième génération, explique qu'il est pour lui primordial d'utiliser l'humour plutôt que le pathos ou les clichés victimaires pour transmettre la mémoire de la Shoah. L'humour empêche qu'une commémoration devienne une mise en scène de l'horreur et donc un reflet du nazisme. De plus, connaissant l'aversion du parti nazi pour l'humour, la possibilité de rire permet d'éviter de concéder une victoire de plus à l'adversaire.

Rire, mémoire, Shoah conclut sur l'étude du cinéma des années 1990 avec d'abord La liste de Schindler (1993) de Steven Spielberg, et ensuite des comédies de la Shoah : La vie est belle (1997) de Roberto Benigni, Train de vie (1998) de Radu Mihaileanu et Jakob le menteur (1999) de Peter Kassovitz.

Pour Sébastien Fevry, spécialiste de la comédie cinématographique, les trois derniers films prennent leur sens justement après la sortie du film de Spielberg. $\mathrm{La}$ liste de Schindler faisait office de référence que les comédies subséquentes s'affairaient à déconstruire. Ces comédies tendent à favoriser une mémoire du bien qui rejoint l'Histoire et contraste avec le mal historique souvent représenté. Pour ce faire, elles introduisent des éléments incongrus dans le fil du récit, ce qui suscite chez l'auditeur dérouté une réflexion plus poussée sur ce qu'il vient de voir. La fable permet aussi une approche plus douce de la Catastrophe pour ceux qui n'y seraient pas initiés, comme les enfants : "L'évasion rêvée vers un autre monde permet de tenir provisoirement le réel à distance et d'y revenir par la suite, mieux armé pour l'affronter» (p. 326).

Pour Lauterwein - comme elle l'exprime dans son article « Nouvelles ingénuités. La vie est belle ? " (p. 349-366) -, la réussite de films comme celui de Roberto Benigni n'est toutefois pas aussi évidente. Selon l'auteure, La vie est belle ne traite pas précisément de la Shoah, mais témoigne plutôt de notre tendance à nous dérober face à la réalité de l'horreur, ce qui explique justement son grand succès. L'auditeur se conforterait dans cette version tronquée de la Catastrophe tout en croyant avoir participé à la transmission de la mémoire. Selon Lauterwein, la catharsis provoquée par ces comédies de la Shoah est détournée par un désir cinématographique d'innover en matière de représentation et par le sentiment du devoir accompli (en ayant contribué à la transmission de la mémoire) trop rapidement perçu par le public.

$$
* * *
$$

Rire, mémoire, Shoah tente de montrer comment les vertus du rire sont infinies et intemporelles, quant à la transmission d'une mémoire aussi lourde que celle du massacre de près de six millions de Juifs. La Shoah est prise en exemple, mais il va de soi que bon nombre de ces réflexions s'appliquent à tout génocide, quel qu'il soit. 
Comprendre comment les victimes et les écrivains ont pu s'aider du rire pour survivre avant, pendant ou après la guerre permet une approche différente des conflits passés, présents ou à venir, et force le lecteur à réinterpréter la connaissance qu'il en a.

En misant sur 25 auteurs aux spécialités diverses, l'ouvrage collectif permet d'offrir une vue d'ensemble sur la question. C'est un pas énorme vers une meilleure compréhension des bienfaits du rire en temps de crise. De plus, traiter du rire comme moyen de transmission de la mémoire, c'est au fond participer soi-même à cette transmission, tout en triant les éléments mémoriaux du passé pour les générations futures et, en ce sens, l'entreprise de Lauterwein est plus que salutaire.

Bien que les textes ne fassent jamais référence l'un à l'autre, leur disposition permet au lecteur de bien saisir comment les auteurs se confrontent ou se répondent. Certaines précisions manquent, toutefois, par exemple lorsqu'il faut séparer l'humour juif de l'humour non juif. Le lecteur est souvent laissé à lui-même pour faire cette distinction très importante puisque toutes les victimes du nazisme n'étaient pas juives. Pour l'aider dans cette tâche, il aurait été intéressant d'avoir un point de comparaison avec une œuvre portant sur un autre conflit que la Shoah pour fournir un aperçu des attributs universels du rire. En 2016 néanmoins, plusieurs populations ressentent les effets d'un génocide, passé ou présent, et l'analyse réalisée par Rire, mémoire, Shoah nous donne plusieurs pistes pour aider ou comprendre les victimes.

Si le rire était tout ce qui restait d'humain à certains détenus, prisonniers depuis d'interminables mois ou années dans les pires conditions imaginables, c'est qu'en effet, comme le dit Gengis Cohn, "le rire est le propre de l'homme » (p. 38). Utilisons-le alors... mais avec goût! 